\title{
Wahrgenommene Wertschätzung im Lehramtsstudium
}

\author{
Fachunterschiede und Effekte auf Wohlbefinden \\ und Abbruchsintention
}

\author{
Bastian Carstensen ${ }^{1}$ (), Christoph Lindner ${ }^{2}$ und Uta Klusmann¹ \\ ${ }^{1} I P N$ - Leibniz-Institut für die Pädagogik der Naturwissenschaften und Mathematik, Kiel, Deutschland \\ ${ }^{2}$ Universität Hamburg, Deutschland
}

\begin{abstract}
Zusammenfassung: Die wahrgenommene Wertschätzung stellt nicht nur im Arbeits- sondern auch im Hochschulkontext eine wichtige soziale Ressource für die akademische Leistung, das Engagement und Wohlbefinden dar. Studentinnen und Studenten, die sich in ihren Lehrveranstaltungen wertgeschätzt fühlen, lernen erfolgreicher und neigen seltener zu Studienabbruchsintentionen. Erste Untersuchungen zeigen, dass Studentinnen und Studenten mit dem Abschlussziel Lehramt sich im Vergleich zu Fachstudentinnen und -studenten weniger von ihren Dozentinnen und Dozenten wertgeschätzt fühlen, allerdings kontrollieren diese Studien nicht für intraindividuelle Unterschiede zwischen den Studienfächern. Vor diesem Hintergrund haben wir untersucht, wie sich $N=1255$ Lehramtsstudentinnen und -studenten in ihren zwei Studienfächern hinsichtlich ihrer Kompetenzen wertgeschätzt fühlen und welche personenbezogenen und fachspezifischen Faktoren die wahrgenommene Wertschätzung beeinflussen. Die Ergebnisse der mehrebenanalytischen Modelle zeigten, dass die wahrgenommene Wertschätzung durch die Dozentinnen und Dozenten vor allem auf Ebene der studierten Fächer variierte, wobei in der MINT-Fächergruppe weniger Wertschätzung wahrgenommen wurde. Zweitens haben wir anhand einer längsschnittlichen Substichprobe $(N=436)$ untersucht, inwieweit die wahrgenommene Wertschätzung mit Indikatoren des Wohlbefindens, der Studienabbruchsintention sowie Veränderungen in diesen Outcomes im Verlauf eines Jahres assoziiert ist. Anhand der Ergebnisse zeigte sich, dass Studentinnen und Studenten, die sich mehr wertgeschätzt fühlten, auch zufriedener mit ihrem Studium und ihren Lehrveranstaltungen waren, mehr Fachenthusiasmus berichteten, weniger erschöpft waren und seltener zu Studienabbruchsintentionen neigten. Zusätzlich war die wahrgenommene Wertschätzung mit der Veränderung in diesen Outcomes assoziiert. Die Befunde werden vor dem Hintergrund praktischer Problemfelder wie dem Lehrkräftemangel diskutiert.
\end{abstract}

Schlüsselwörter: Wertschätzung, Lehramtsstudium, Wohlbefinden, Studienabbruchsintentionen

\section{Perceived Apprecation in University Teacher Education: Subject Differences and Effects on Well-Being and Intention to Quit}

\begin{abstract}
Perceived appreciation is an important social resource for academic performance, engagement and well-being in both work and higher education contexts. Students who feel valued by their lecturers learn more successfully and tend to drop out of college less often. Research has already shown that teacher candidates feel less valued by their lecturers compared to students in other subject areas. However, these studies did not control for intra-individual differences between subjects. Against this background, we examined how $N=1255$ teacher candidates feel appreciated with regard to their competencies by the lecturers in their two subjects of study. Moreover, we investigated which personal and subject-specific factors affect this perception of appreciation. Multi-level analyses showed that the perceived appreciation varies mainly at the subject level, with teacher candidates in STEM subjects feeling less valued. On the basis of a longitudinal subsample ( $N=436)$ we further investigated how the teacher candidates' perceived appreciation is associated with indicators of well-being, intention to drop out of college, and changes in these outcomes over the course of one year. According to the results, students who felt more appreciated by their lecturers were also more satisfied with their studies and courses, reported more subject enthusiasm, were less exhausted and were less inclined to drop out of college. In addition, students who felt appreciated developed more favorably with regard to these outcomes. We discuss our findings in the light of practice-related problem areas such as teacher shortage.
\end{abstract}

Keywords: Appreciation, University Teacher Education, Well-Being, Intention to Quit

Die subjektiv empfundene Wertschätzung geht in Studium und Beruf mit positiven Konsequenzen für Wohlbefinden und Leistungsfähigkeit einher (Bakker, Hakanen, Demerouti \& Xanthopoulou, 2007; Gavish \& Friedman, 2010; Pfister et al., 2020; Schneider \& Preckel, 2017). Die Berufsgruppe der Lehrkräfte fühlt sich seitens der Gesell- schaft wenig wertgeschätzt (Eurydice, 2004; Pinn \& Rothland, 2011; Rothland, 2016). Bereits Lehramtsstudentinnen und -studenten werden geringere Kompetenzen zugeschrieben als Studentinnen und Studenten in anderen Feldern (Ihme \& Möller, 2015), obwohl empirische Befunde aufzeigen, dass keine Negativselektion in das 
Lehramtsstudium erfolgt (Roloff Henoch, Klusmann, Lüdtke \& Trautwein, 2015). Zudem berichten Studentinnen und Studenten aus dem Lehramt, dass sie sich in den fachwissenschaftlichen Lehrveranstaltungen, die sie in der Regel gemeinsam mit Fachstudentinnen und -studenten besuchen, von ihren Dozentinnen und Dozenten abgewertet fühlen (Erdmann \& Ratzlaff, 2017).

Dabei können die Folgen einer als gering empfundenen Wertschätzung weitreichend sein. Ein Mangel an wahrgenommener Wertschätzung der eigenen Kompetenzen kann zu (Selbst-)Stereotypen führen, die wiederum einen negativen Effekt auf die Leistungsfähigkeit haben (vgl. stereotype threat; Ihme \& Möller, 2015). Laut Gómez Tutor, Hobelsberger und Menzer (2011) spielt die Wertschätzung seitens der Dozentinnen und Dozenten für die Studentenschaft eine zentrale Rolle hinsichtlich des Studienerfolgs. Wahrgenommene Defizite in der Wertschätzung erhöhen hingegen die Studienabbruchsintentionen (Deuer, 2017). Dies erscheint vor dem Hintergrund des akuten und prognostizierten Lehrkräftemangels - v. a. in den mathematisch-naturwissenschaftlichen Fächern (Klemm, 2015; Kultusministerkonferenz [KMK], 2020) - als problematisch.

Trotz erster Hinweise darauf, dass Lehramtsstudentinnen und -studenten sich weniger wertgeschätzt fühlen als ihre Kommilitoninnen und Kommilitonen im Fachstudium (Erdmann \& Ratzlaff, 2017) bleibt bisher unklar, wie stark diese Wahrnehmung möglicherweise innerhalb des Lehramts zwischen verschiedenen Fächern bzw. Fächergruppen variiert. Zudem wurden die Folgen der wahrgenommenen Wertschätzung für Studentinnen und Studenten bisher nur im Querschnitt betrachtet. Vor diesem Hintergrund untersucht die vorliegende Studie Fach- und Personenmerkmale, die mit der wahrgenommenen Wertschätzung im Lehramtsstudium zusammenhängen. Zudem betrachten wir, welche Konsequenzen die wahrgenommene Wertschätzung wiederum auf verschiedene Indikatoren des Wohlbefindens und die Studienabbruchsintention sowie die Entwicklung in diesen Kriteriumsvariablen über den Zeitraum eines Jahres hat.

\section{Wertschätzung als wichtige Ressource für Erfolg und Wohlbefinden}

Im Kontext arbeits- und organisationspsychologischer Arbeiten wird Wertschätzung als Anerkennung der individuellen Eigenschaften einer Person sowie ihrer Kompetenzen und Leistungen definiert (Pfister et al., 2020). Sie drückt sich beispielsweise über Rückmeldungen wie Lob oder konstruktives Feedback (Stocker, Jacobshagen, Krings, Pfister \& Semmer, 2014; Yukl, Gordon \& Taber, 2002) aus und kommuniziert Fürsorge, aufrichtiges Interesse und Respekt (Beehr \& Glazer, 2001; Stocker et al., 2019; van Quaquebeke \& Felps, 2016). Zumeist wird die wahrgenommene Wertschätzung in der Forschung als Teil von breit angelegten psychologischen Konstrukten behandelt (vgl. Pfister et al., 2020; Stocker et al., 2019). Beispielsweise lässt sich Wertschätzung der emotionalen Komponente sozialer Unterstützung zuordnen, die im Rahmen der Stress- und Belastungsforschung eine zentrale Umweltressource darstellt (vgl. Halbesleben, 2006; Kinman, Wray \& Strange, 2011; Viswesvaran, Sanchez \& Fisher, 1999). Auch beschreibt das Modell beruflicher Gratifikationskrisen (effort-reward-imbalance-model; Siegrist, 1996), das die Genese von Stress im Arbeitskontext erklärt, die Wertschätzung als wichtige Ressource für das berufliche Wohlbefinden. Berufliches Wohlbefinden wird häufig in Form einer kognitiven und einer affektiven Komponente beschrieben. Die kognitive Komponente beinhaltet u.a. die Berufszufriedenheit und die affektive Komponente das berufliche Engagement und die Freude an der beruflichen Tätigkeit (Diener, Suh, Lucas \& Smith, 1999).

Empirische Arbeiten zeigten den Stellenwert der Wertschätzung für das berufliche Wohlbefinden bereits in mehreren Kontexten. So fanden Stocker, Jacobshagen, Semmer und Annen (2010) in einer Querschnittstudie positive Zusammenhänge zwischen der wahrgenommenen Wertschätzung und der Berufszufriedenheit von Soldaten. Zusätzlich zeigte sich in dieser Studie, dass die erfahrene Wertschätzung den negativen Zusammenhang zwischen der Anzahl der Arbeitsstunden und Berufszufriedenheit verringerte (Stocker et al., 2010). Eine Tagebuchstudie mit Angestellten in verschiedenen Berufen (u.a. Krankenpflege) ergab zudem, dass sich das Erleben wertschätzender Interaktionen (z.B. ausgesprochenes Lob, Dankbarkeit) auf das tägliche Wohlbefinden zum Feierabend auswirkte (Stocker et al., 2014). Schließlich zeigten Pfister et al. (2020), dass die empfundene Wertschätzung - u.a. operationalisiert durch erfahrene Aufmerksamkeit, Dankbarkeit und dem Verständnis durch Vorgesetzte und Kolleginnen und Kollegen - einen positiven Einfluss auf das emotionale Erleben im Beruf hatte.

Auch bei Lehrkräften wurde die wahrgenommene Wertschätzung bereits als eine wichtige Ressource für das berufliche Wohlbefinden im Sinne von Zufriedenheit und Engagement identifiziert (Bakker et al., 2007; Gavish \& Friedman, 2010). Im Rahmen einer Untersuchung zum Wohlbefinden von Berufseinsteigerinnen und Berufseinsteigern im Lehrerberuf fanden Gavish und Friedman (2010), dass sowohl die durch die unterrichteten Schülerinnen und Schüler wahrgenommene Wertschätzung als auch die durch die Gesellschaft wahrgenommene Wert- 
schätzung für Lehrkräfte im ersten Berufsjahr negativ mit den Burnout-Symptomen emotionaler Erschöpfung, Depersonalisation und reduzierter persönlicher Leistungsfähigkeit assoziiert war. Bakker et al. (2007) untersuchten bei Lehrkräften den Zusammenhang von Ressourcen, Stressoren und dem beruflichen Engagement und zeigten, dass die Wertschätzung durch Kolleginnen und Kollegen insbesondere unter schwierigen Bedingungen verglichen mit anderen Ressourcen den stärksten Prädiktor für die Arbeitsbereitschaft, wahrgenommene Sinnhaftigkeit der beruflichen Tätigkeiten und Konzentration darstelle.

Für den Hochschulkontext zeigte ein systematisches Review, dass die Wahrnehmung wertschätzenden Verhaltens seitens der Dozentinnen und Dozenten deutlich mit der Studienleistung assoziiert war $(d=0.47$; vgl. Schneider \& Preckel, 2017). Des Weiteren sprechen die Ergebnisse von Befragungen an Hochschulen für den hohen Stellenwert wahrgenommener Wertschätzung in Bezug auf Studienerfolg (Gómez Tutor et al., 2011) und Studienabbruchsintention (Deuer, 2017). Im Rahmen einer Untersuchung von Gratifikationskrisen im dualen Studium deckte Deuer (2017) für Studentinnen und Studenten unterschiedlicher Studienbereiche eine starke Assoziation zwischen wahrgenommener Wertschätzung und Studienabbruchsintentionen auf. Knapp die Hälfte der Befragten, die angaben sich eher bzw. voll und ganz wertgeschätzt zu fühlen wies keinerlei Abbruchtendenz auf, wohingegen eine als gering wahrgenommene Wertschätzung (eher bzw. überhaupt keine Zustimmung) bei etwa jeder fünften befragten Person mit einer hohen Studienabbruchsintention einherging.

Insgesamt weisen die bisherigen empirischen Befunde darauf hin, dass die wahrgenommene Wertschätzung im Rahmen der beruflichen Tätigkeiten eine Ressource für das berufliche Wohlbefinden darstellt. Insbesondere Lehrkräfte scheinen davon zu profitieren, seitens der Gesellschaft, ihrer Kolleginnen und Kollegen sowie ihrer Schülerinnen und Schüler Wertschätzung zu erfahren. Im Hochschulkontext zeigen Studentinnen und Studenten, die sich wertgeschätzt fühlen, bessere Studienleistungen und neigen seltener zu Abbruchsintentionen. Für die Beziehung zwischen wahrgenommener Wertschätzung und ihrem Wohlbefinden liegen hingegen bisher keine Befunde vor.

\section{Wahrgenommene Wertschätzung im Lehramtsstudium: Empirische Befunde}

Pinn und Rothland (2011) stellten fest, dass Lehramtsstudentinnen und -studenten in den Medien deutlich ver- nehmbar abgewertet werden und schlussfolgerten einen großen Einfluss der Berichterstattung auf die öffentliche Meinung gegenüber den Nachwuchslehrkräften. Unterstützung findet diese Schlussfolgerung durch die Befunde von Ihme und Möller (2015) zur Annahme (negativer) Stereotype. Sie befragten Studentinnen und Studenten abseits des Lehramts, berufstätige bzw. arbeitssuchende Personen sowie Schülerinnen und Schüler zu ihrem Bild über Lehramtsstudentinnen und -studenten. Im Vergleich mit Studentinnen und Studenten der Fächer Jura, Informatik und Psychologie schrieben die Befragten den Lehramtsstudentinnen und -studenten im Sinne des Stereotype Content Model (Fiske, Cuddy, Glick \& Xu, 2002) geringere Kompetenzen, aber eine stärker ausgeprägte Warmherzigkeit zu. Dies deckte sich mit der Selbstwahrnehmung von Studentinnen und Studenten mit dem Abschlussziel Lehramt, die ebenfalls berichteten, dass sie von ihren Kommilitoninnen und Kommilitonen außerhalb des Lehramts als weniger kompetent, aber warmherzig wahrgenommen werden (Ihme \& Möller, 2015). Roloff Henoch et al. (2015) zeigten, dass Lehramtsstudentinnen und Studenten tatsächlich extravertierter und sozial interessierter waren als ihre Kommilitoninnen und Kommilitonen im Fachstudium; die Annahme geringerer kognitiver Voraussetzungen von Lehramtsstudentinnen und Studenten im Vergleich zu Studentinnen und Studenten anderer Studienprofile wurde hingegen nicht bestätigt.

Die Befunde von Ihme und Möller (2015) weisen darauf hin, dass das negative Bild über Lehramtsanwärterinnen und -anwärter hinsichtlich einer geringen Leistungsfähigkeit bzw. wenig Kompetenzen von unterschiedlichen Personengruppen geteilt wird - selbst von den Lehramtsstudentinnen und -studenten. Einige Hinweise darauf, dass auch die Dozentinnen und Dozenten an Hochschulen die Kompetenzen ihrer Studentengruppen unterschiedlich einschätzen, ergeben sich aus einer qualitativ angelegten Studie mit dem Lehrpersonal im Fach Mathematik. Günther und Koeszegi (2015) werteten die Beiträge von Diskussionsrunden aus und fanden, dass sich die Mathematikdozentinnen und -dozenten von anderen Fachdisziplinen distanzierten und diesen häufig negative Eigenschaften zuschrieben. Studentinnen und Studenten mit dem Abschlussziel Lehramt wurde weniger Begabung für das Fach Mathematik attestiert, die zum einen durch eine Selbstselektion bei der Studienwahl und zum anderen durch die durch das Lehramtscurriculum begrenzte Zeitkapazität bedingt sei. Ferner ergaben sich aus den Diskussionen Hinweise darauf, dass die Ungleichbehandlung von Studentinnen und Studenten unterschiedlicher Abschlussprofile im wissenschaftlichen Feld verbreitet ist. Studentinnen und Studenten aus MINT-Fächern schätzen ihr Studium im Vergleich zu Studentinnen und Studenten 
anderer Fachrichtungen (z.B. Sozial- und Sprachwissenschaften) als schwieriger ein, sodass die wahrgenommenen Studienanforderungen im MINT-Bereich häufiger die selbstzugeschriebenen Bewältigungskompetenzen übersteigen (Fellenberg \& Hannover, 2006). Vorurteile der Dozentinnen und Dozenten gegenüber Lehramtsstudentinnen und -studenten in den MINT-Fächern, wie sie sich in der Studie von Günther und Koeszegi (2015) beispielhaft für das Fach Mathematik gezeigt haben, können diese Wahrnehmung womöglich verstärken. Vorurteile gegenüber Frauen, die insbesondere in den MINT-Fächern auftreten (vgl. Ertl, Luttenberger \& Paechter, 2014; Münst, 2008), wurden in den Diskussionen hingegen nicht explizit thematisiert. Während Günther und Koeszegi (2015) die Perspektive der Hochschullehrerinnen und -lehrer beleuchteten, widmeten sich Erdmann und Ratzlaff (2017) der Wahrnehmung von Studentinnen und Studenten im Kontext ihrer universitären Lehrveranstaltungen. Ergebnisse ihrer qualitativen Befragung zum sozialen Klima zeigten, dass einige Lehramtsstudentinnen und -studenten sich durch ihre Dozentinnen und Dozenten abgewertet fühlten. Diese gaben beispielsweise an, „als weniger gut ausgebildete Wissenschaftler angesehen [zu werden], die in den höheren Studienbereichen nichts zu suchen haben“ (Erdmann \& Ratzlaff, 2017, S.188). Gleichzeitig ergab sich kein statistisch signifikanter Unterschied zu Fachstudentinnen und -studenten hinsichtlich der Häufigkeit von Schwierigkeiten im Umgang mit dem Lehrpersonal (Erdmann \& Ratzlaff, 2017). Dieser Vergleich erfolgte allerdings ohne die Berücksichtigung möglicher Effekte von Studienfächern oder Personencharakteristika.

Insgesamt lässt die Befundlage zur Wertschätzung gegenüber Lehramtsstudentinnen und -studenten noch einige Fragen offen. Zwar weisen erste Studien darauf hin, dass die (negativen) Stereotype gegenüber dieser Studentendengruppe (vgl. Ihme \& Möller, 2015) auch von Dozentinnen und Dozenten geteilt werden. So zeigen die Ergebnisse von Günther und Koeszegi (2015), dass Studentinnen und Studenten im Lehramt im Vergleich zu anderen Studienprofilen als eher weniger begabt eingeschätzt werden. Allerdings wurde in dieser Studie lediglich die Perspektive aus dem Fach Mathematik berücksichtigt, was keine Generalisierung auf alle lehramtsbildenden Studienfächer ermöglicht. Des Weiteren wurde noch nicht ausreichend untersucht, ob die potenziellen Vorurteile der Dozentinnen und Dozenten verhaltenswirksam werden. Erste Hinweise liefert die Studie von Erdmann und Ratzlaff (2017) zum sozialen Klima in universitären Lehrveranstaltungen. Die Ergebnisse zeigen, dass Lehramtsstudentinnen und -studenten durchaus Defizite in der Wertschätzung seitens ihrer Dozentinnen und Dozenten wahrnehmen. Dass in dieser Studie nicht für individuelle Personenmerkmale wie das Geschlecht oder Kontextmerkmale der Studien- fächer (z.B. Fächergruppe, Anzahl Lehramtsstudentinnen und -studenten im Fach) kontrolliert wurde, schränkt die Aussagekraft dieser Ergebnisse allerdings ein.

\section{Die aktuelle Studie}

Die dargestellten empirischen Befunde verdeutlichen, dass die wahrgenommene Wertschätzung im Arbeitskontext und im Studium - etwa durch Vorgesetzte, Kolleginnen und Kollegen oder die Dozentinnen und Dozenten eine wichtige Ressource darstellt, die mit Aspekten des Wohlbefindens, dem Engagement und der Leistung assoziiert ist. Hochschullehrerinnen und -lehrer scheinen der Gruppe von Studentinnen und Studenten mit dem Abschlussziel Lehramt im Vergleich zu Fachstudentinnen und -studenten geringere Kompetenzen zuzuschreiben, was von dieser Gruppe wiederum als abwertend bzw. wenig wertschätzend erlebt werden kann. Wie stark die wahrgenommene Wertschätzung zwischen Fächern und Personen variiert und inwiefern fachspezifische Faktoren und individuelle Personencharakteristika diese Variation erklären können, lässt sich aus den bisherigen Befunden hingegen nicht schlussfolgern. Zudem fehlt es an Studien, die die Zusammenhänge zum Wohlbefinden und zur Studienabbruchsintention längsschnittlich untersuchen.

Im Zuge der ersten Forschungsfrage der vorliegenden Studie beschäftigten wir uns damit, inwieweit Lehramtsstudentinnen und -studenten sich in ihren fachwissenschaftlichen Lehrveranstaltungen, die sie gemeinsam mit Fachstudentinnen und -studenten besuchen, wertgeschätzt fühlen. Hierzu wurde das mittlere Niveau in der wahrgenommenen Wertschätzung durch die Dozentinnen und Dozenten betrachtet, wobei die Angaben sich vornehmlich auf die durch die Dozentinnen und Dozenten zugeschriebene Leistungsfähigkeit bzw. Kompetenz bezogen. Ferner untersuchten wir die Variation zwischen den beiden Studienfächern sowie zwischen den Studentinnen und Studenten und betrachteten Zusammenhänge mit Fachspezifika sowie den individuellen Personencharakteristika. Die Ergebnisse von Günther und Koeszegi (2015) deuten darauf hin, dass Lehramtsstudentinnen und -studenten in den mathematisch-naturwissenschaftlichen Fächern tendenziell eine geringere Wertschätzung berichten. Daher erwarteten wir, dass die empfundene Wertschätzung in den MINT-Fächern geringer ausfällt (Hypothese 1a). Um Kompositionseffekte in den Studienfächern zu untersuchen, bezogen wir zusätzlich den Zusammenhang zwischen der wahrgenommenen Wertschätzung und dem relativen Anteil der Studentinnen und Studenten mit dem Abschlussziel Lehramt im jeweiligen Fach ein. Wir hatten die Annahme, dass Vorurteile der Dozentin- 


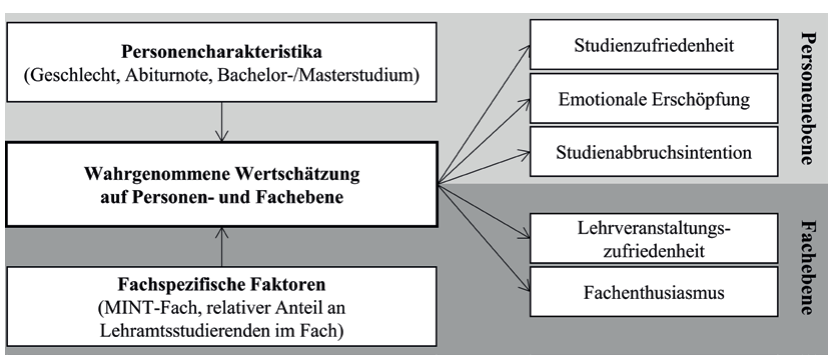

Abbildung 1. Heuristisches Arbeitsmodell der vorliegenden Studie. Im Rahmen der ersten Forschungsfrage wird die von Lehramtsstudentinnen und -studenten wahrgenommene Wertschätzung in Abhängigkeit von Kontext- und individuellen Merkmalen betrachtet. Die zweite Forschungsfrage konzentriert sich auf die Vorhersage mehrerer Aspekte des Wohlbefindens und der Studienabbruchsintention durch die wahrgenommene Wertschätzung.

nen und Dozenten gegenüber der Leistungsfähigkeit von Lehramtsstudentinnen und -studenten (vgl. Günther \& Koeszegi, 2015) seltener in Studienfächern auftreten, in denen diese Gruppe als Majorität vertreten ist. Entsprechend gingen wir davon aus, dass der relative Anteil dieser Studentengruppe im Fach positiv mit der empfundenen Wertschätzung in Zusammenhang steht (Hypothese $1 \mathrm{~b}$ ). Der Zusammenhang zwischen den individuellen Merkmalen Geschlecht, Abiturnote, Studienstatus (Bachelor/ Master) und der empfundenen Wertschätzung wurde aufgrund fehlender theoretisch begründbarer Hypothesen explorativ untersucht.

Die zweite Forschungsfrage der vorliegenden Studie betraf die Rolle der wahrgenommenen Wertschätzung für das subjektive Wohlbefinden und die Abbruchsintention im Lehramtsstudium. Gemäß den theoretischen Annahmen aus der Stress- und Belastungsforschung (z.B. effort-reward-imbalance-model; Siegrist, 1996) erwarteten wir, dass die wahrgenommene Wertschätzung eine Ressource darstellt und somit positiv mit dem subjektiven Wohlbefinden assoziiert ist. Gleichzeitig nahmen wir einen negativen Zusammenhang zwischen wahrgenommener Wertschätzung und der Abbruchsintention an. Als Indikatoren des subjektiven Wohlbefindens nutzten wir Angaben zur Zufriedenheit mit den fachwissenschaftlichen Lehrveranstaltungen und dem Fachenthusiasmus, die für beide Studienfächer getrennt erfragt wurden, sowie fachübergreifende Angaben zur Studienzufriedenheit und zur emotionalen Erschöpfung im Studium.

Während die Relevanz wertschätzender Verhaltensweisen seitens der Dozentinnen und Dozenten für die Studienleistung bereits aufgezeigt wurde (Schneider \&
Preckel, 2017), fokussierte sich diese Studie somit auf den Zusammenhang der wahrgenommenen Wertschätzung mit dem subjektiven Wohlbefinden und der Studienabbruchsintention. Die konkreten Hypothesen zur zweiten Forschungsfrage lauteten wie folgt: Die wahrgenommene Wertschätzung ist ein signifikanter Prädiktor für die Zufriedenheit in den fachwissenschaftlichen Lehrveranstaltungen (Hypothese 2a), den Fachenthusiasmus (Hypothese 2b) und die allgemeine Studienzufriedenheit (Hypothese 3a). Ferner weisen Studentinnen und Studenten, die sich in ihren Lehrveranstaltungen wertgeschätzt fühlen, eine geringere emotionale Erschöpfung (Hypothese $3 b$ ) sowie weniger Abbruchsintentionen (Hypothese 3c) auf. Abbildung 1 zeigt das heuristische Arbeitsmodell zur vorliegenden Studie und bietet eine Übersicht zu den auf Personen- und Fachebene untersuchten Zusammenhängen.

\section{Methode}

\section{Stichprobe und Vorgehen}

Die Untersuchung der vorliegenden Fragestellungen basierte auf Daten der Student Teacher Professional Development Study (STePS ${ }^{1}$; vgl. Carstensen \& Klusmann, 2021; Lindner \& Klusmann, 2018), die mittels jährlicher Erhebungen an einer norddeutschen Universität die professionelle Entwicklung zukünftiger Lehrkräfte im Studium verfolgt. $\mathrm{Zu}$ jedem Messzeitpunkt werden alle für ein Lehramtsstudium eingeschriebenen Studentinnen und Studenten der Universität kontaktiert und dazu eingeladen, die Online-Umfrage zu bearbeiten. Für die aktuelle Studie wurden die Daten zweier Messzeitpunkte aus STePS in den Jahren 2017 und 2018 verwendet (Lindner, Klusmann, Baum, Brouër, Burda-Zoyke et al., 2018; Lindner, Klusmann, Baum, Brouër, Heinz et al., 2018).

Die Untersuchung der ersten Forschungsfrage unserer Studie erfolgte unter Einbezug des zweiten Messzeitpunkts aus dem Projekt im Jahr 2018. Die Gesamtstichprobe zur Umfrage im Jahr 2018 umfasste $N=1255$ Lehramtsstudentinnen und -studenten, was einer Ausschöpfungsquote von $28.6 \%$ aller für das Lehramt eingeschriebenen Studentinnen und Studenten am untersuchten Universitätsstandort entspricht. Die Befragten waren überwiegend weiblich $(67 \%)$ und durchschnittlich $23.72(S D=3.43)$ Jahre alt. Die durchschnittliche Note der Hochschulzugangsberechtigung lag bei $2.28(S D=0.52) .42 \%$ der Befragten studierten mindestens ein Fach, das sich der MINT-Fächergruppe ${ }^{2}$

\footnotetext{
Die Student Teacher Professional Development Study ist Teil der Qualitätsoffensive Lehrerbildung, die seit 2015 vom Bundesministerium für Bildung und Forschung (BMBF) gefördert wird.

Als MINT-Fächer berücksichtigt wurden: Biologie, Chemie, Geographie, Informatik, Mathematik und Physik.
} 
zuordnen lässt. Zum Erhebungszeitpunkt hatten 26\% der Befragten bereits das Bachelorstudium abgeschlossen und befanden sich im Masterstudium. Statistische Vergleiche zwischen der Studentenpopulation an der untersuchten Universität und unserer Stichprobe ergaben keine eindeutigen Hinweise auf einen Selektionsbias, allerdings waren Studentinnen leicht überrepräsentiert $(p<.001, V=.08)$.

Für die zweite Fragestellung wurde der erste Messzeitpunkt des Projekts (2017) in die Analysen einbezogen, um das Ausgangsniveau der Teilnehmerinnen und Teilnehmer hinsichtlich ihres Wohlbefindens und ihrer Abbruchsintention statistisch berücksichtigen zu können. Aus der Gesamtstichprobe hatten $N=436$ Studentinnen und Studenten bereits an der Umfrage im Vorjahr teilgenommen. In der für den Längsschnitt berücksichtigten Stichprobe war das Durchschnittsalter etwas höher und es waren mehr Studentinnen und Studenten aus dem Master vertreten. Ferner nahmen mehr Studentinnen im Längsschnitt teil und die Abiturnote war im Durchschnitt leicht besser. Der Anteil von Studentinnen und Studenten mit mindestens einem MINT-Fach unterschied sich nicht zur Gesamtstichprobe. Weitere Informationen und Kennwerte zu den Selektivitätsanalysen sind in Tabelle E1 im elektronischen Supplement (ESM 1) dargestellt.

\section{Instrumente}

Um Zusammenhänge auf Personen- und auf Fachebene differenziert betrachten zu können, machten die Befragten im Rahmen der STePS-Umfrage sowohl getrennte Angaben für das Erst- und Zweitfach als auch allgemeine Angaben mit Bezug auf das Lehramtsstudium als Ganzes. Sofern nicht anders beschrieben, wurden die Einschätzungen auf 4-stufigen Likert-Skalen ( $1=$ trifft überhaupt nicht $z u, 4=$ trifft völlig $z u$ ) vorgenommen. Die vollständigen Skalen können in Tabelle E2 im ESM 1 eingesehen werden.

\section{Fachspezifische Angaben}

Wahrgenommene Wertschätzung. Inwieweit die Lehramtsstudentinnen und -studenten sich durch ihre Dozentinnen und Dozenten in den fachwissenschaftlichen Lehrveranstaltungen wertgeschätzt fühlen, schätzten sie für jedes ihrer zwei Studienfächer anhand von fünf Items (adaptiert nach Renger, Renger, Miché \& Simon, 2017) ein. Die Skala beinhaltete eine allgemeine Einschätzung („In den fachwissenschaftlichen Lehrveranstaltungen in meinem Hauptfach X werden Lehramtsstudierende von den Lehrenden nach meiner Wahrnehmung und Erfahrung ebenso wertgeschätzt wie Fachstudierende") sowie spezifische Angaben zur wahrgenommenen leistungsbasierten Wertschätzung (z.B. „[...] geben mir die Lehrenden zu verste- hen, dass sie mich für einen leistungsfähigen Studierenden halten"). Die Skala zeigte für beide Fächer eine hohe Reliabilität $\left(.83 \leq \alpha_{\mathrm{T} 1} \leq .85\right)$.

Fachenthusiasmus. Die Erfassung des Fachenthusiasmus erfolgte in Anlehnung an die Skala von Kunter, Frenzel, Nagy, Baumert und Pekrun (2011). Sie umfasste drei Items, die sich auf die persönlich empfundene Wichtigkeit des Fachs und die Begeisterung für das Fach beziehen (z.B. „Ich finde dieses Fach spannend“). Sowohl zum ersten Messzeitpunkt $\left(.82 \leq \alpha_{\mathrm{T} 1} \leq .89\right)$ als auch zum zweiten Messzeitpunkt $\left(.86 \leq \alpha_{\mathrm{T} 2} \leq .88\right)$ ergaben sich gute Werte für die interne Konsistenz.

Lehrveranstaltungszufriedenheit. Die Zufriedenheit der Lehramtsstudentinnen und -studenten mit ihren Lehrveranstaltungen wurde anhand von drei angepassten Items der Skala von Westermann, Elke, Spies und Trautwein (1996) erfasst (z. B. „Insgesamt bin ich mit den Lehrveranstaltungen in diesem Fach zufrieden"). Die Reliabilität der Skala war zu beiden Messzeitpunkten gut $\left(.87 \leq \alpha_{\mathrm{T} 1} \leq .90\right.$; $.84 \leq \alpha_{\mathrm{T} 2} \leq .89$ ).

\section{Allgemeine Angaben}

Studienzufriedenheit. Die Erfassung der allgemeinen Studienzufriedenheit erfolgte analog zur fachspezifisch erhobenen Lehrveranstaltungszufriedenheit mittels adaptierter Items von Westermann et al. (1996). Die Lehramtsstudentinnen und -studenten bewerteten die Aussagen von drei Items (z. B. „Insgesamt bin ich mit meinem jetzigen Studium zufrieden"). Die interne Konsistenz war hoch $\left(\alpha_{\mathrm{T} 1}=.80\right.$, $\left.\alpha_{\mathrm{T} 2}=.85\right)$.

Emotionale Erschöpfung. Das Erleben emotionaler Erschöpfung im Lehramtsstudium wurde anhand von vier Items der deutschen Version (Enzmann \& Kleiber, 1989) des Maslach Burnout Inventars (Maslach, Jackson \& Leiter, 1996) erfasst (z.B. „Ich fühle mich in meinem Studium oft erschöpft"). Die Skala erwies sich zu beiden Messzeitpunkten als reliabel $\left(\alpha_{\mathrm{T} 1}=.80, \alpha_{\mathrm{T} 2}=.82\right)$.

Studienabbruchsintention. Die Intention zum Studienabbruch wurde mit drei Items erfasst (z. B. „Ich habe schon öfter daran gedacht, das Lehramtsstudium abzubrechen“; vgl. Blüthmann, Thiel und Wolfgramm, 2011; Fellenberg \& Hannover, 2006). Die aus den Items gebildete Skala zeigte eine gute Reliabilität $\left(\alpha_{\mathrm{T} 1}=.76, \alpha_{\mathrm{T} 2}=.82\right)$.

\section{Kontextvariablen}

Um die jeweilige Situation der Teilnehmerinnen und Teilnehmer umfassend einzuschätzen, wurden neben den genannten Konstrukten mehrere Kontextvariablen in die Analysen der vorliegenden Studie einbezogen. Erstens wurden Angaben der Lehramtsstudentinnen und -studenten zu ihrem aktuell angestrebten Studienabschluss genutzt, d.h. ob sie sich aktuell im Bachelor- oder bereits im Masterstudium befanden. Zweitens wurde die Fächer- 
gruppe berücksichtigt, wobei wir für die aktuelle Studie zwischen Fächern aus dem MINT-Bereich und allen weiteren Fächern unterschieden haben. Die Fächergruppe wurde in einer Dummy-Variable kodiert ( 0 = kein MINT-Fach, $1=$ MINT-Fach). Drittens wurde eine Variable zur relativen Häufigkeit der Studentinnen und Studenten mit dem Abschlussziel Lehramt im Fach berechnet. Diese Variable beschreibt den relativen Anteil von Lehramtsstudentinnen und -studenten im jeweiligen Fach, indem ihre Anzahl durch die Gesamtzahl der Studentinnen und Studenten in Fach- und Lehramtsstudium geteilt wurde. Die fachspezifischen Immatrikulationszahlen wurden uns vom Studierendensekretariat der Universität zur Verfügung gestellt. Für das Fach Mathematik ergab sich im Bachelorstudium beispielsweise ein Wert von $h=0.63$, d.h. $63 \%$ der Studentinnen und Studenten im Bachelor mit dem Fach Mathematik hatten Lehramt als Abschlussziel (vgl. Tabelle E3 im ESM 1). In diesem Beispiel stellten die Lehramtsstudentinnen und -studenten also eine Majorität und die Fachstudentinnen und -studenten eine Minorität dar. Je größer der Wert der Variable $\left(h_{\text {Min }}=0 ; h_{\text {Max }}=1\right)$, auf desto mehr Studentinnen und Studenten des Lehramts - und weniger Fachstudentinnen und -studenten - trifft die jeweilige Person in ihren fachwissenschaftlichen Lehrveranstaltungen.

\section{Statistische Analysen}

Sämtliche in der vorliegenden Studie untersuchten Hypothesen wurden im Vorfeld der Datenanalyse präregistriert (Carstensen, Lindner \& Klusmann, 20213). Die Hypothesen zur ersten Forschungsfrage bezüglich der Zusammenhänge empfundener Wertschätzung mit fachspezifischen Faktoren und den individuellen Personencharakteristika wurden mit Mehrebenen-Regressionsanalysen untersucht. Die Modellierung mehrerer Ebenen ermöglicht die Berücksichtigung der geschachtelten Struktur in den Daten (Fächer in Personen). Auf diese Weise kann nicht nur für Abhängigkeiten in den Daten kontrolliert werden, was Verzerrungen in den Schätzungen vorbeugt (vgl. Nezlek, Schröder-Abé \& Schütz, 2006). Vielmehr lässt die spezifische Analyse der Personen- und Fachebene zu, die Effekte für jede Ebene getrennt zu interpretieren. Die für die erste Forschungsfrage spezifizierten Modelle enthielten die empfundene Wertschätzung als abhängige Variable sowie Prädiktorvariablen auf Level 1 (innerhalb der Person, z. B. die Fächergruppe) und Level 2 (zwischen Personen, z. B. das Geschlecht). Die Untersuchung der Forschungsfrage hinsichtlich der Zusammenhänge zwischen wahrgenommener Wertschätzung und dem Wohlbefinden sowie der
Abbruchsintention erfolgte ebenfalls unter Berücksichtigung der Mehrebenenstruktur. Zuerst wurde unter Berücksichtigung der Fach- und Personenvariablen lediglich der Zusammenhang zwischen Wertschätzung und dem jeweiligen Kriterium geschätzt. Anschließend wurde die Kriteriumsvariable aus dem Vorjahr als Prädiktor in das Modell aufgenommen, um zu testen, ob die wahrgenommene Wertschätzung Veränderungen in dieser Variable vorhersagen kann. Die fachspezifischen Angaben zur Lehrveranstaltungszufriedenheit und zum Fachenthusiasmus wurden auf Fach- und Personenebene ausgewertet. Die Studienzufriedenheit, die emotionale Erschöpfung sowie die Abbruchsintention, welche sich generell auf das Studium bezogen und somit nur einmal pro Person vorlagen, wurden nur auf Personenebene ausgewertet. Die Analysen zur Beantwortung der zweiten Forschungsfrage basierten auf $N=436$ Studentinnen und Studenten, die zu beiden Messzeitpunkten an der Umfrage teilgenommen haben.

Die statistischen Analysen wurden mit Mplus 7.4 (Muthén \& Muthén, 1998-2015) durchgeführt. Zur besseren Interpretierbarkeit der Ergebnisse wurden alle Variablen, ausgenommen der dichotomen Variablen, vor den Analysen z-standardisiert $(M=0, S D=1)$. Eine Überprüfung des Messmodells mittels konfirmatorischer Faktorenanalyse deutete auf eine gute psychometrische Qualität der eingesetzten Skalen hin (RMSEA $=.06, \mathrm{CFI}=.92$, $\mathrm{TLI}=.90$ ); die Mehrebenen-Regressionsanalysen wurden zur Reduzierung der Komplexität manifest durchgeführt. Fehlende Werte (1-3\%) wurden mit dem Full-Information-Maximum-Likelihood-Verfahren (FIML) geschätzt (Enders, 2010).

\section{Ergebnisse}

\section{Wahrgenommene Wertschätzung im Lehramtsstudium: Zusammenhänge auf Personen- und Fachebene}

Das erste Ziel der vorliegenden Studie war es, die von Lehramtsstudentinnen und -studenten durch ihre Dozentinnen und Dozenten wahrgenommene Wertschätzung und ihre Zusammenhänge mit personenbezogenen und fachspezifischen Faktoren zu untersuchen. Der Personenmittelwert für die wahrgenommene Wertschätzung lag bei $M=3.17\left(S D_{\text {Fach }}=0.55 ; S D_{\text {Person }}=0.34\right)$, also deutlich oberhalb des theoretischen Skalenmittelwerts und weist darauf hin, dass die befragten Studentinnen und Studenten sich in ihren beiden Studienfächern im 
Tabelle 1. Deskriptive Statistiken und Korrelationen auf Personen- (Level $2\left[L_{2}\right]$, oberhalb der diagonale) und Fachebene (Level $1\left[L_{1}\right]$, unterhalb der Diagonale) für die Gesamtstichprobe ( $N=1255)$

\begin{tabular}{|c|c|c|c|c|c|c|c|c|c|c|c|c|c|c|}
\hline & & M & $S D$ & 1 & 2 & 3 & 4 & 5 & 6 & 7 & 8 & 9 & 10 & 11 \\
\hline 1 & Geschlecht ${ }^{a}\left(L_{2}\right)$ & 0.33 & 0.47 & & .07 & -.02 & -.03 & -.04 & .05 & .16 & -.26 & -.01 & -.06 & -.04 \\
\hline 2 & Abiturnote $\left(L_{2}\right)$ & 2.28 & 0.53 & & & -.04 & -.07 & .06 & .03 & -.28 & -.03 & -.06 & -.11 & -.25 \\
\hline 3 & Studienabschluss ${ }^{\mathrm{b}}\left(\mathrm{L}_{2}\right)$ & 0.28 & 0.44 & & & & -.01 & -.08 & -.09 & -.14 & .62 & .02 & .13 & .23 \\
\hline 4 & Studienzufriedenheit $\left(\mathrm{L}_{2}\right)$ & 3.15 & 0.58 & & & & & -.51 & -.47 & -.04 & .02 & .42 & .92 & .89 \\
\hline 5 & Emotionale Erschöpfung $\left(\mathrm{L}_{2}\right)$ & 2.32 & 0.70 & & & & & & -.40 & .18 & -.16 & -.44 & -.62 & -.42 \\
\hline 6 & Abbruchsintention $\left(\mathrm{L}_{2}\right)$ & 1.44 & 0.63 & & & & & & & -.01 & -.06 & -.21 & -.44 & -.51 \\
\hline 7 & MINT-Fach ${ }^{\circ}\left(\mathrm{L}_{1}\right)$ & 0.28 & 0.45 & & & & & & & & -.82 & -.66 & -.22 & .05 \\
\hline 8 & Rel. Anteil Lehramt $\left(\mathrm{L}_{1}\right)$ & 0.62 & 0.17 & & & & & & & -.52 & & .54 & .24 & .11 \\
\hline 9 & Wertschätzung $\left(\mathrm{L}_{1}\right)$ & 3.17 & 0.64 & & & & & & & -.43 & .33 & & .68 & .45 \\
\hline 10 & Lehrveranstaltungszufriedenheit $\left(L_{1}\right)$ & 3.01 & 0.69 & & & & & & & -.12 & .03 & .35 & & .92 \\
\hline 11 & Fachenthusiasmus $\left(\mathrm{L}_{1}\right)$ & 3.43 & 0.62 & & & & & & & -.05 & -.06 & .10 & .46 & \\
\hline
\end{tabular}

Anmerkungen: ${ }^{\mathrm{a}} 0=$ weiblich, $1=$ männlich. ${ }^{\mathrm{b}} 0=$ Bachelor, $1=$ Master. ${ }^{\mathrm{c}} 0=$ andere Fächergruppe, $1=$ MINT-Fach. Antwortformat aller Skalen: $1=$ trifft überhaupt nicht zu, 4 = trifft völlig zu; statistisch signifikante Korrelationen $(p<.05)$ sind fettgedruckt.

Durchschnitt eher wertgeschätzt fühlten. Die Intraklassenkorrelation der empfundenen Wertschätzung betrug $I C C_{(1)}=0.31$, was bedeutet, dass $31 \%$ der Variation in der wahrgenommenen Wertschätzung auf Unterschiede zwischen den Personen zurückzuführen sind. Mit 69\% lag ein Großteil der Varianz allerdings auf Fachebene, was gegen eine generelle Bewertungstendenz der Lehramtsstudentinnen und -studenten spricht und darauf hinweist, dass die Wertschätzung durch die Dozentinnen und Dozenten in Erst- und Zweitfach differenziert betrachtet wurde. Tabelle 1 fasst die deskriptiven Statistiken der untersuchten Variablen auf Fach- und Personenebene zusammen.

Die Ergebnisse der Mehrebenen-Regressionsmodelle, die wir zur Untersuchung der zur ersten Forschungsfrage formulierten Hypothesen spezifiziert haben, sind in Tabelle 2 zusammengefasst. Im Rahmen der ersten Hypothese (Hypothese 1a) überprüften wir, ob die Fächergruppe signifikant mit der wahrgenommenen Wertschätzung assoziiert ist. Das zugehörige Regressionsmodell (Modell 1) beinhaltete auf Fachebene die studierte Fächergruppe, welche signifikant mit der empfundenen Wertschätzung assoziiert war $(B=-0.90, p<.001)$. Dies bedeutet, dass sich die Lehramtsstudentinnen und -studenten hypothesenkonform in den MINT-Fächern weniger wertgeschätzt fühlten. Die erklärte Varianz auf Fachebene belief sich in diesem Modell auf 22\%. Zur Überprüfung unserer zweiten Hypothese (Hypothese 1b) spezifizierten wir ein Modell, in dem der relative Anteil der Lehramtsstudentinnen und -studenten im Fach als Prädiktor berücksichtigt wurde. Dieser Anteil war signifikant mit der wahrgenommenen Wertschätzung assoziiert $(B=0.31$, $p<.001)$ und klärte $13.5 \%$ der Varianz auf Fachebene auf.
Wie angenommen empfanden Lehramtsstudentinnen und -studenten, wenn sie einen größeren relativen Anteil im Fach ausmachten, mehr Wertschätzung durch ihre Dozentinnen und Dozenten. Das dritte Modell berücksichtigte die Fächergruppe und den relativen Anteil von Lehramtsstudentinnen und -studenten in den Fächern gemeinsam als Prädiktoren. In diesem Modell waren sowohl die Fächergruppe $(B=-0.74, p<.001)$ als auch die Zusammensetzung der Studentinnen und Studenten $(B=0.19$,

Tabelle 2. Vorhersage der wahrgenommenen Wertschätzung durch Personenvariablen sowie Merkmale der studierten Fächer

\begin{tabular}{|c|c|c|c|}
\hline & Modell 1 & Modell 2 & Modell 3 \\
\hline & $B(S E)$ & $B(S E)$ & $B(S E)$ \\
\hline Intercept & $0.24(0.03)$ & $0.03(0.03)$ & $0.22(0.03)$ \\
\hline \multicolumn{4}{|l|}{ Personenebene $\left(L_{2}\right)$} \\
\hline Geschlecht $^{a}$ & $0.09(0.05)$ & $0.08(0.05)$ & $0.12(0.05)$ \\
\hline Abiturnote & $-0.11(0.02)$ & $-0.03(0.02)$ & $-0.10(0.02)$ \\
\hline Studienstatus ${ }^{b}$ & $-0.07(0.05)$ & $-0.21(0.05)$ & $-0.19(0.05)$ \\
\hline \multicolumn{4}{|l|}{ Fachebene $\left(L_{1}\right)$} \\
\hline MINT-Fach ${ }^{\circ}$ & $-0.90(0.05)$ & & $-0.74(0.05)$ \\
\hline $\begin{array}{l}\text { Relativer Anteil } \\
\text { Lehramtsabschluss }\end{array}$ & & $0.31(0.02)$ & $0.19(0.02)$ \\
\hline \multicolumn{4}{|l|}{$R^{2}$} \\
\hline Personenebene & .04 & .04 & .06 \\
\hline Fachebene & .22 & .14 & .27 \\
\hline
\end{tabular}

Anmerkungen: ${ }^{\mathrm{a}} 0=$ weiblich, $1=$ männlich. ${ }^{\mathrm{b}} 0=$ Bachelor, $1=$ Master ${ }^{\circ} 0=$ andere Fächergruppe, $1=$ MINT-Fach. Unstandardisierte Regressionsgewichte der z-standardisierten Variablen, Werte in Klammern kennzeichnen die Standardfehler; statistisch signifikante Koeffizienten $(p<.05)$ sind fettgedruckt. 
$p<.001)$ signifikant mit der wahrgenommenen Wertschätzung assoziiert. Gemeinsam klärten diese fachspezifischen Prädiktoren 27\% der Varianz in den Angaben zur Wertschätzung auf.

Die explorativ untersuchten Zusammenhänge zwischen wahrgenommener Wertschätzung und den berücksichtigten Personenvariablen waren in den Modellen unterschiedlich stark akzentuiert (vgl. Tabelle 2). Unter gemeinsamer Berücksichtigung der Prädiktoren auf Fachebene (Modell 3) waren alle berücksichtigten Personenvariablen signifikant mit der wahrgenommenen Wertschätzung assoziiert. Männliche Lehramtsstudenten empfanden tendenziell mehr Wertschätzung als ihre Kommilitoninnen ( $B=0.12, p=.009)$, ebenso wie Studentinnen und Studenten mit besserer Abiturnote $(B=-0.10, p<.001)$ und Studentinnen und Studenten im Bachelorstudium $(B=-0.19$, $p<.001)$. Insgesamt erklärten diese Prädiktoren $6 \%$ der Varianz auf Personenebene.

\section{Vorhersage fachspezifischer und allge- meiner Indikatoren des Wohlbefindens durch die wahrgenommene Wertschätzung}

Zur Untersuchung des Zusammenhangs von wahrgenommener Wertschätzung und dem Wohlbefinden haben wir unter anderem ihre Effekte auf die fachspezifischen Angaben zur Lehrveranstaltungszufriedenheit und zum Fachenthusiasmus analysiert. Die Modellergebnisse sind in Tabelle 3 darstellt.

Die wahrgenommene Wertschätzung war signifikant mit der Zufriedenheit in den fachwissenschaftlichen Lehrveranstaltungen sowohl auf Personenebene $(B=0.38$, $p=.001)$ als auch auf Fachebene $(B=0.55, p<.001)$ assoziiert. Hingegen war der Fachenthusiasmus lediglich auf Fachebene signifikant mit der wahrgenommenen Wertschätzung assoziiert $(B=0.25, p<.001)$. Das bedeutet, dass Studentinnen und Studenten, die sich durch ihre Do-

Tabelle 3. Vorhersage des Wohlbefindens und der Abbruchsintention durch die wahrgenommene Wertschätzung.

\begin{tabular}{|c|c|c|c|c|c|c|c|c|c|c|}
\hline & \multicolumn{2}{|c|}{$\begin{array}{l}\text { Lehrveranstal- } \\
\text { tungszufriedenheit }\end{array}$} & \multicolumn{2}{|c|}{ Fachenthusiasmus } & \multicolumn{2}{|c|}{$\begin{array}{c}\text { Studien- } \\
\text { zufriedenheit }\end{array}$} & \multicolumn{2}{|c|}{$\begin{array}{l}\text { Emotionale } \\
\text { Erschöpfung }\end{array}$} & \multicolumn{2}{|c|}{ Abbruchsintentior } \\
\hline & M1 & M2 & M1 & M2 & M1 & M2 & M1 & M2 & M1 & M2 \\
\hline & $B(S E)$ & $B(S E)$ & $B(S E)$ & $B(S E)$ & $B(S E)$ & $B(S E)$ & $B(S E)$ & $B(S E)$ & $B(S E)$ & $B(S E)$ \\
\hline Intercept & $\begin{array}{c}-0.29 \\
(0.10)\end{array}$ & $\begin{array}{c}-0.22 \\
(0.12)\end{array}$ & $\begin{array}{c}-0.42 \\
(0.11)\end{array}$ & $\begin{array}{l}-0.29 \\
(0.11)\end{array}$ & $\begin{array}{l}0.10 \\
(0.15)\end{array}$ & $\begin{array}{l}-0.06 \\
(0.13)\end{array}$ & $\begin{array}{l}0.30 \\
(0.15)\end{array}$ & $\begin{array}{c}0.32 \\
(0.12)\end{array}$ & $\begin{array}{l}0.31 \\
(0.15)\end{array}$ & $\begin{array}{l}0.36 \\
(0.12)\end{array}$ \\
\hline \multicolumn{11}{|l|}{ Personenebene $\left(L_{2}\right)$} \\
\hline Wertschätzung & $\begin{array}{l}0.38 \\
(0.12)\end{array}$ & $\begin{array}{c}0.12 \\
(0.13)\end{array}$ & $\begin{array}{c}0.14 \\
(0.13)\end{array}$ & $\begin{array}{c}0.05 \\
(0.12)\end{array}$ & $\begin{array}{c}0.98 \\
(0.21)\end{array}$ & $\begin{array}{c}0.69 \\
(0.18)\end{array}$ & $\begin{array}{r}-0.93 \\
(0.22)\end{array}$ & $\begin{array}{r}-0.68 \\
(0.18)\end{array}$ & $\begin{array}{c}-0.52 \\
(0.18)\end{array}$ & $\begin{array}{r}-0.30 \\
(0.14)\end{array}$ \\
\hline Baseline $^{a}$ & & $\begin{array}{l}1.15 \\
(0.24)\end{array}$ & & $\begin{array}{c}0.63 \\
(0.23)\end{array}$ & & $\begin{array}{c}0.49 \\
(0.06)\end{array}$ & & $\begin{array}{r}0.49 \\
(0.06)\end{array}$ & & $\begin{array}{c}0.58 \\
(0.05)\end{array}$ \\
\hline Geschlecht ${ }^{b}$ & $\begin{array}{c}-0.02 \\
(0.08)\end{array}$ & $\begin{array}{c}-0.06 \\
(0.08)\end{array}$ & $\begin{array}{c}-0.07 \\
(0.08)\end{array}$ & $\begin{array}{c}-0.02 \\
(0.08)\end{array}$ & $\begin{array}{c}0.04 \\
(0.11)\end{array}$ & $\begin{array}{c}0.08 \\
(0.09)\end{array}$ & $\begin{array}{r}-0.18^{\dagger} \\
(0.10)\end{array}$ & $\begin{array}{r}-0.16^{\dagger} \\
(0.08)\end{array}$ & $\begin{array}{l}0.02 \\
(0.11)\end{array}$ & $\begin{array}{r}-0.01 \\
(0.09)\end{array}$ \\
\hline Abiturnote & $\begin{array}{r}-0.08 \\
(0.03)\end{array}$ & $\begin{array}{c}0.01 \\
(0.04)\end{array}$ & $\begin{array}{c}-0.05 \\
(0.04)\end{array}$ & $\begin{array}{c}0.01 \\
(0.04)\end{array}$ & $\begin{array}{l}-0.07 \\
(0.05)\end{array}$ & $\begin{array}{l}-0.07 \\
(0.04)\end{array}$ & $\begin{array}{c}0.01 \\
(0.05)\end{array}$ & $\begin{array}{c}0.01 \\
(0.04)\end{array}$ & $\begin{array}{c}-0.05 \\
(0.05)\end{array}$ & $\begin{array}{c}0.01 \\
(0.04)\end{array}$ \\
\hline Studienstatus ${ }^{\circ}$ & $\begin{array}{c}0.16 \\
(0.07)\end{array}$ & $\begin{array}{c}0.12 \\
(0.07)\end{array}$ & $\begin{array}{l}0.27 \\
(0.07)\end{array}$ & $\begin{array}{c}0.17 \\
(0.07)\end{array}$ & $\begin{array}{c}-0.08 \\
(0.10)\end{array}$ & $\begin{array}{c}0.03 \\
(0.08)\end{array}$ & $\begin{array}{r}-0.18^{+} \\
(0.10)\end{array}$ & $\begin{array}{r}-0.20 \\
(0.08)\end{array}$ & $\begin{array}{c}-0.23 \\
(0.09)\end{array}$ & $\begin{array}{r}-0.26 \\
(0.07)\end{array}$ \\
\hline \multicolumn{11}{|l|}{ Fachebene $\left(L_{1}\right)$} \\
\hline Wertschätzung & $\begin{array}{c}0.55 \\
(0.06)\end{array}$ & $\begin{array}{l}0.45 \\
(0.06)\end{array}$ & $\begin{array}{c}0.25 \\
(0.07)\end{array}$ & $\begin{array}{c}0.21 \\
(0.06)\end{array}$ & & & & & & \\
\hline MINT-Fach & $\begin{array}{c}0.28 \\
(0.09)\end{array}$ & $\begin{array}{c}0.25 \\
(0.08)\end{array}$ & $\begin{array}{c}0.28 \\
(0.10)\end{array}$ & $\begin{array}{c}0.25 \\
(0.09)\end{array}$ & & & & & & \\
\hline $\begin{array}{l}\text { Relativer Anteil } \\
\text { Lehramtsabschluss }\end{array}$ & $\begin{array}{c}-0.14 \\
(0.04)\end{array}$ & $\begin{array}{c}-0.13 \\
(0.21)\end{array}$ & $\begin{array}{c}-0.12 \\
(0.04)\end{array}$ & $\begin{array}{r}-0.09 \\
(0.04)\end{array}$ & & & & & & \\
\hline Baseline & & $\begin{array}{c}0.33 \\
(0.04)\end{array}$ & & $\begin{array}{c}0.39 \\
(0.06)\end{array}$ & & & & & & \\
\hline \multicolumn{11}{|l|}{$R^{2}$} \\
\hline Personenebene & .53 & .91 & .33 & .75 & .28 & .50 & .26 & .49 & .09 & .42 \\
\hline Fachebene & .18 & .29 & .04 & .18 & & & & & & \\
\hline
\end{tabular}

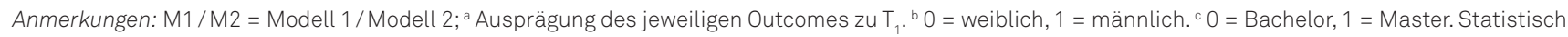
signifikante Koeffizienten $(p<.05)$ sind fettgedruckt; ${ }^{\dagger} p<.10$. 
zentinnen und Dozenten wertgeschätzt fühlten, insgesamt zufriedener mit ihren Lehrveranstaltungen im Studium waren. Zudem trug die im jeweiligen Fach empfundene Wertschätzung direkt zur Lehrveranstaltungszufriedenheit und zum Enthusiasmus in diesem Fach bei. Auch die Fächergruppe war signifikant mit der Lehrveranstaltungszufriedenheit $(B=0.28, p<.002)$ und dem Fachenthusiasmus assoziiert $(B=0.28, p=.007)$. Somit standen diese Indikatoren des Wohlbefindens unter statistischer Kontrolle der wahrgenommenen Wertschätzung und der Zusammensetzung im Fach mit der MINT-Fächergruppe in positivem Zusammenhang. Studentinnen und Studenten in der MINT-Fächergruppe waren demnach dann zufriedener und enthusiastischer, wenn sie sich durchschnittlich wertgeschätzt fühlten und der relative Anteil von Studentinnen und Studenten mit Lehramtsabschluss im Fach durchschnittlich ausfiel. Der relative Anteil an Lehramtsstudentinnen und -studenten im Fach zeigte hingegen einen leicht negativen Zusammenhang mit der Lehrveranstaltungszufriedenheit $(B=-0.14, p<.001)$ und dem Fachenthusiasmus $(B=-0.12, p<.001)$. Schließlich waren Masterstudentinnen und -studenten sowohl zufriedener mit ihren fachwissenschaftlichen Lehrveranstaltungen $(B=0.16$, $p=.025)$ als auch enthusiastischer $(B=0.27, p<.001)$. In einem zweiten Schritt (Modell 2) haben wir untersucht, inwiefern die wahrgenommene Wertschätzung einen Effekt auf die Veränderungen des Wohlbefindens hat und dafür die Werte der Kriteriumsvariablen aus dem Vorjahr in die Analysen aufgenommen. Auf der Personenebene zeigten sich keine statistisch signifikanten Zusammenhänge zwischen der wahrgenommenen Wertschätzung und den Veränderungen in der Lehrveranstaltungszufriedenheit und dem Fachenthusiasmus. Auf Ebene der Fächer ging eine höhere wahrgenommene Wertschätzung in einem Fach auch mit einem statistisch signifikanten Zuwachs in der Lehrveranstaltungszufriedenheit $(B=0.45, p<.001)$ und dem Fachenthusiasmus $(B=0.21, p=.001)$ einher. Studentinnen und Studenten beurteilten die Zufriedenheit und ihren Enthusiasmus in ihrem Studienfach somit höher als im Vorjahr, wenn sie sich aktuell von den Dozentinnen und Dozenten wertgeschätzt fühlten. Diese Ergebnisse stützen unsere Annahmen über die Zusammenhänge der wahrgenommenen Wertschätzung mit der Zufriedenheit in den fachwissenschaftlichen Lehrveranstaltungen (Hypothese 2a) und dem Fachenthusiasmus (Hypothese 2b).

Während die Angaben zur Lehrveranstaltungszufriedenheit und dem Fachenthusiasmus jeweils fachspezifisch vorlagen, wurden die weiteren Indikatoren des Wohlbefindens-Studienzufriedenheit und emotionale Erschöpfungfachübergreifend erhoben, ebenso die Studienabbruchsintention. Entsprechend wurden diese Kriteriumsvariablen nur auf Personenebene (Level 2) spezifiziert. Die Angaben der empfundenen Wertschätzung wurden zum Zwecke der Berücksichtigung ihrer between- und within-Anteile hingegen weiterhin auf Fach- und Personenebene spezifiziert (vgl. Asparouhov \& Muthén, 2019). Tabelle 3 zeigt die Modellparameter für die Vorhersage der fachübergreifend erhobenen Kriteriumsvariablen.

Die zum zweiten Messzeitpunkt erhobenen Kriteriumsvariablen Studienzufriedenheit $(B=0.98, p<.001)$, emotionale Erschöpfung $(B=-0.93, p<.001)$ sowie Studienabbruchsintention $(B=-0.52, p=.004)$ wurden jeweils signifikant durch die empfundene Wertschätzung vorhergesagt. Die von den Studentinnen und Studenten wahrgenommene Wertschätzung durch ihre Dozentinnen und Dozenten leistete demnach einen maßgeblichen Beitrag zu ihrem Wohlbefinden im Studium. Darüber hinaus machten sich Studentinnen und Studenten, die sich in ihren Lehrveranstaltungen wertgeschätzt fühlten, weniger Gedanken um einen Studienabbruch. Zusätzlich berichteten die Studentinnen und Studenten im Master geringere Abbruchsintentionen $(B=-0.23, p=.015)$ als im Bachelorstudium. Auch unter Berücksichtigung der Ausgangswerte in den Kriteriumsvariablen blieben die Zusammenhänge zur wahrgenommenen Wertschätzung statistisch signifikant (Studienzufriedenheit: $B=0.69, p<.001$; emotionale Erschöpfung: $B=-0.68, p<.001$; Abbruchsintention: $B=-0.30, p=.031$ ), was bedeutet, dass Studentinnen und Studenten mit höherer subjektiver Wertschätzung gleichzeitig auch eine positive Veränderung in ihrem Wohlbefinden erlebten und die Abbruchsintention sank. Zudem berichteten Studentinnen und Studenten im Master unter Kontrolle der Werte aus dem Vorjahr eine geringere emotionale Erschöpfung $(B=-0.20, p<.015)$ und weniger Abbruchsintentionen $(B=-0.26, p<.001)$. Insgesamt bestätigen die Ergebnisse unsere Hypothesen zu den Zusammenhängen zwischen der wahrgenommenen Wertschätzung und der Studienzufriedenheit, der emotionalen Erschöpfung (Hypothesen $3 \mathrm{a} \& 3 \mathrm{~b}$ ) sowie der Studienabbruchsintention (Hypothese $3 \mathrm{c}$ ).

\section{Zusammenfassung und Diskussion}

In der vorliegenden Studie sollte untersucht werden, inwieweit sich Studentinnen und Studenten mit dem Abschlussziel Lehramt von ihren Dozentinnen und Dozenten wertgeschätzt fühlen, wie die wahrgenommene Wertschätzung mit personen- und fachspezifischen Faktoren zusammenhängt und welche Effekte sie auf das Wohlbefinden sowie die Studienabbruchsintention hat. Im Einklang mit unseren Hypothesen zeigten sich fachspezifische Unterschiede in der wahrgenommenen Wertschätzung. Des Weiteren war die wahrgenommene Wertschätzung mit mehreren Indikatoren des Wohlbefindens 
und der Studienabbruchsintention assoziiert. Im Folgenden werden die Ergebnisse im Detail betrachtet.

Insgesamt war das mittlere Niveau der wahrgenommenen Wertschätzung durch die Dozentinnen und Dozenten relativ hoch, was tendenziell gegen generell abwertende Verhaltensweisen von ihrer Seite gegenüber den Studentinnen und Studenten im Lehramt spricht, wie es beispielsweise bei Erdmann und Ratzlaff (2017) angedeutet worden ist. Die Betrachtung der wahrgenommenen Wertschätzung in Abhängigkeit von personen- und fachspezifischen Faktoren geschah in der vorliegenden Studie über einen mehrebenenanalytischen Ansatz, der uns eine getrennte Interpretation von personen- und fachspezifischen Zusammenhängen ermöglichte. Die Mehrebenenanalysen ergaben, dass über zwei Drittel der Variation in den Angaben zur wahrgenommenen Wertschätzung auf die konkreten Studienfächer einer Person zurückzuführen sind und sprechen somit gegen eine personenspezifische Bewertungstendenz. Im Einklang mit unserer ersten Hypothese war die wahrgenommene Wertschätzung in den MINT-Fächern deutlich niedriger ausgeprägt als in den anderen Fächergruppen; der Effekt betrug ca. eine Standardabweichung. Eine Ursache für diesen bedeutsamen Unterschied liegt womöglich in den Vorurteilen hinsichtlich der Begabung von Lehramtsstudentinnen und -studenten seitens der Dozentinnen und Dozenten in den MINT-Fächern (vgl. Günther \& Koeszegi, 2015 für das Fach Mathematik). Möglicherweise führen auch geschlechtsspezifische Vorurteile (vgl. Bian, Leslie \& Cimpian, 2018) zu einer geringeren Wertschätzung gegenüber Studentinnen und Studenten des Lehramts, da Frauen in dieser Gruppe mehrheitlich vertreten sind. Dies geht zumindest teilweise mit unserem Befund einher, dass männliche Studenten sich mehr wertgeschätzt fühlten als ihre Kommilitoninnen. Dieser Zusammenhang zeigte sich allerdings unabhängig vom Studienfach bzw. der Fächergruppe. Die wahrgenommene Wertschätzung war neben der Fächergruppe auch mit dem relativen Anteil von Lehramtsstudentinnen und -studenten im Fach assoziiert. Im Einklang mit unserer zweiten Hypothese bedeutet dies, dass sich diese Gruppe eher in den Fächern wertgeschätzt fühlt, in denen sie mehrheitlich vertreten ist. Möglicherweise stellen sich die Dozentinnen und Dozenten in diesen Fächern eher auf Lehramtsstudentinnen und -studenten ein, indem sie beispielsweise häufiger einen Schulbezug herstellen und damit den spezifischen Interessen und Bedarfen zukünftiger Lehrkräfte entgegenkommen, was einer Facette von Wertschätzung entspricht (Beehr \& Glazer, 2001; Yukl et al., 2002).

Neben dem Geschlecht waren auch die weiteren explorativ untersuchten Personenfaktoren mit der wahrgenommenen Wertschätzung assoziiert. Eine schlechtere Abiturnote ging mit einer als geringer wahrgenommenen
Wertschätzung einher. Da eine schlechtere Abiturnote in der Regel mit schlechteren Studienleistungen einhergeht (vgl. Blömeke, 2009; Trapmann, Hell, Weigand \& Schuler, 2007) ist es denkbar, dass hier die leistungsbezogene Anerkennung seitens der Dozentinnen und Dozenten geringer ausfällt. Schließlich fühlten sich die Lehramtsstudentinnen und -studenten im Master weniger wertgeschätzt als jene im Bachelorstudium. Womöglich führt der Umfang der fachbezogenen Lerngelegenheiten, der im Lehramtsstudium im Gegensatz zum Fachstudium geringer ausfällt, im fortgeschrittenen Studium zu unterschiedlichen Leistungsniveaus, sodass insbesondere die Lehramtsstudentinnen und -studenten im Master weniger leistungsbezogene Anerkennung von ihren Dozentinnen und Dozenten erhalten.

Im Rahmen der zweiten Fragestellung untersuchten wir die Relevanz der wahrgenommenen Wertschätzung für mehrere Indikatoren des Wohlbefindens und die Studienabbruchsintention. Hypothesenkonform zeigten die Ergebnisse, dass die wahrgenommene Wertschätzung einen bedeutsamen Prädiktor für die Studien- und Lehrveranstaltungszufriedenheit, den Fachenthusiasmus sowie die emotionale Erschöpfung darstellt. Auch im Längsschnitt, d.h. unter Kontrolle der Ausgangswerte in den Kriteriumsvariablen, blieb dieses Befundmuster bestehen. Die wahrgenommene Wertschätzung durch die Dozentinnen und Dozenten ist somit nicht nur mit dem absoluten Niveau des Wohlbefindens, sondern auch mit dessen Entwicklung assoziiert. Damit bestätigen diese Ergebnisse die für den Arbeitskontext bereits empirisch gezeigten Befunde zur Relevanz der wahrgenommenen Wertschätzung für das berufliche Wohlbefinden (u.a. Pfister et al., 2020) auch für die Gruppe von Studentinnen und Studenten, die ein Lehramt anstreben. Die berücksichtigten Indikatoren des Wohlbefindens verdeutlichen, dass die wahrgenommene Wertschätzung sich nicht nur in der unmittelbaren Zufriedenheit mit den jeweiligen Lehrveranstaltungen niederschlägt, sondern auch den Fachenthusiasmus, d.h. die Begeisterung und das Interesse für das Fach nachhaltig beeinflusst. Dieser stellt einen wichtigen Faktor für den späteren beruflichen Erfolg als Lehrkraft dar (Frenzel, Goetz, Lüdtke, Pekrun \& Sutton, 2009; Kunter et al., 2008). Schließlich zeigt der Zusammenhang zwischen wahrgenommener Wertschätzung und emotionaler Erschöpfung nochmals den hohen Stellenwert auf, den Studentinnen und Studenten dem Verhalten ihrer Dozentinnen und Dozenten zuschreiben.

Analog zu den Indikatoren des Wohlbefindens untersuchten wir den Zusammenhang zwischen der wahrgenommenen Wertschätzung durch die Dozentinnen und Dozenten und der Studienabbruchsintention, wobei wahrgenommene Defizite in der Wertschätzung mit einer höheren Studienabbruchsintention einhergingen (vgl. Deuer, 2017). Auch 
die intraindividuellen Veränderungen in der Studienabbruchsintention über ein Jahr waren mit der wahrgenommenen Wertschätzung assoziiert. Dieser Befund ergänzt die Ergebnisse von Isleib, Woisch und Heublein (2019), die eine mangelnde akademische und soziale Integration, welche Überschneidungen zum Wertschätzungskonstrukt aufweist, als eine Ursache des Studienabbruchs identifizierten. Studienabbrüche haben neben den negativen ökonomischen und psychologischen Folgen auf individueller Ebene auch gesellschaftliche Folgen, indem sie u.a. zum akademischen Fachkräftemangel beitragen (vgl. Neugebauer, Heublein \& Daniel, 2019). Vor dem Hintergrund des akuten und prognostizierten Lehrkräftemangels, insbesondere in den mathematisch-naturwissenschaftlichen Fächern Chemie, Informatik, Mathematik und Physik (Klemm, 2015; KMK, 2020), sollten Hochschulen an etwaigen Defiziten in der Wertschätzung gegenüber Lehramtsstudentinnen und -studenten arbeiten, um hiermit assoziierte Studienabbruchsintentionen zu minimieren. Beispielsweise könnten Dozentinnen und Dozenten im Rahmen von hochschuldidaktischen Fortbildungskursen dafür sensibilisiert werden, dass Studentinnen und Studenten sowohl in Hinblick auf ihre Studienleistung als auch ihr Wohlbefinden und den Verbleib im Studium von der ihnen entgegengebrachten Wertschätzung profitieren.

\section{Limitationen und Ausblick}

Bei der Interpretation der Ergebnisse der vorliegenden Studie sollten folgende Limitationen beachtet werden. Erstens beschränken sich unsere Ergebnisse auf die Perspektive der Studentinnen und Studenten im Lehramtsstudium. Im Gegensatz zur Studie von Erdmann und Ratzlaff (2017), die zusätzlich Fachstudentinnen und -studenten befragten, konnten wir daher keine Vergleiche zur wahrgenommenen Wertschätzung aus Sicht von Studentinnen und Studenten außerhalb des Lehramts tätigen. Zukünftige Studien könnten durch eine gemeinsame Analyse der Angaben von Studentinnen und Studenten unterschiedlicher Studienprofile weitere Erkenntnisse zu fachspezifischen Unterschieden in der Interaktion zwischen Dozentinnen und Dozenten und Studentinnen und Studenten erbringen. Zweitens beruhen unsere Analysen ausschließlich auf Selbstberichten. Die Berücksichtigung objektiver Leistungsindikatoren wie Studiennoten (vgl. Schneider \& Preckel, 2017) hätte weitere Aussagen zur prognostischen Validität der wahrgenommenen Wertschätzung für den Studienerfolg ermöglicht. Zusätzlich hätten Informationen über einen tatsächlichen Studienabbruch die Aussagekraft der vorliegenden Studie erweitert, auch wenn die selbstberichtete Studienabbruchsintention ein zuverlässiger Indikator für einen späteren Studienabbruch ist
(z.B. Blüthmann, 2012; Mäkinen, Olkinuora \& Lonka, 2004). Folglich sollten zukünftige Untersuchungen diese objektiven Maße berücksichtigen, einerseits um die Befundlage zur Relevanz der wahrgenommenen Wertschätzung zu erweitern, andererseits um mehr Erkenntnisse zu den Ursachen von Studienabbrüchen zu gewinnen, die bisher mehrheitlich im Querschnitt und retrospektiv untersucht wurden (Neugebauer et al., 2019). Drittens ist anzumerken, dass wir mit unseren Daten hinsichtlich der wahrgenommenen Wertschätzung lediglich die Variation zwischen den Fächern untersuchen konnten und sich somit keine Aussagen darüber treffen lassen, wie groß die Unterschiede zwischen einzelnen Dozentinnen und Dozenten im jeweiligen Fach ist. Zuletzt muss berücksichtigt werden, dass wir neben der wahrgenommenen Wertschätzung keine traditionellen Belastungsfaktoren im Studium wie beispielsweise hohe Studienanforderungen oder finanzielle Probleme (vgl. Isleib et al., 2019; Neugebauer et al., 2019) berücksichtigt haben. Neben dem direkten Effekt zwischen der Wertschätzung im Studium und dem Wohlbefinden ist anzunehmen, dass die wahrgenommene Wertschätzung den Zusammenhang zwischen diesen Belastungsfaktoren und dem Wohlbefinden moderiert (vgl. Stocker et al., 2010).

\section{Fazit}

Insgesamt bestätigt die vorliegende Studie die bisherigen Befunde aus dem Arbeitskontext, indem sie den hohen Stellenwert der wahrgenommenen Wertschätzung für das Wohlbefinden auch für Studentinnen und Studenten an der Universität aufzeigt. Trotz des hohen Niveaus, das im Lehramtsstudium in Bezug auf die wahrgenommene Wertschätzung im Durchschnitt angegeben wurde, erscheint es insbesondere vor dem Hintergrund der identifizierten Zusammenhänge mit dem Wohlbefinden bzw. Studienabbruchsintention und deren Entwicklungen problematisch, dass die sich die Lehramtsstudentinnen und -studenten in den MINT-Fächern deutlich weniger wertgeschätzt fühlen. Bereits aktuell fehlt es an Lehrkräften für den mathematisch-naturwissenschaftlichen Bereich, sodass Studienabbrüchen aufgrund mangelnder Wertschätzung seitens der Dozentinnen und Dozenten umso mehr Bedeutung zukommt.

\section{Elektronisches Supplement (ESM)}

Das elektronische Supplement ist mit der Online-Version dieses Artikels verfügbar unter https://doi.org/10.1024/ 1010-0652/a000337 
ESM 1. Selektivitätsanalysen zwischen der Ausgangsstichprobe und den im Längsschnitt betrachteten Personen (Tabelle E1), Verwendete Skalen mit zugehörigen Items (Tabelle E2), Verteilung der Lehramtsstudierenden auf die lehramtsbildenden Fächer sowie die relativen Anteile der Lehramtsstudierenden in Bezug auf die Gesamtzahl Studierender im jeweiligen Fach (Tabelle E3).

\section{Literatur}

Asparouhov, T. \& Muthén, B. (2019). Latent variable centering of predictors and mediators in multilevel and time-series models. Structural Equation Modeling: A Multidisciplinary Journal, 26(1), 119 - 142. https://doi.org/10.1080/10705511.2018.1511375

Bakker, A. B., Hakanen, J. J., Demerouti, E. \& Xanthopoulou, D. (2007). Job resources boost work engagement, particularly when job demands are high. Journal of Educational Psychology, 99(2), 274-284. https://doi.org/10.1037/0022-0663.99.2.274

Beehr, T. A. \& Glazer, S. (2001). A cultural perspective of social support in relation to occupational success. In P. L. Perrewé \& D. C. Ganster (Eds.), Research in occupational stress and well being: Vol. 1. Exploring theoretical mechanisms and perspectives (pp. 97 - 142). Amsterdam, New York: JAl.

Bian, L., Leslie, S.-J. \& Cimpian, A. (2018). Evidence of bias against girls and women in contexts that emphasize intellectual ability. The American Psychologist, 73(9), 1139-1153. https://doi. org/10.1037/amp0000427

Blömeke, S. (2009). Ausbildungs- und Berufserfolg im Lehramtsstudium im Vergleich zum Diplom-Studium - Zur prognostischen Validität kognitiver und psycho-motivationaler Auswahlkriterien. Zeitschrift für Erziehungswissenschaft, 12, 82-110.

Blüthmann, I., Thiel, F. \& Wolfgramm, C. (2011). Abbruchtendenzen in den Bachelorstudiengängen. Individuelle Schwierigkeiten oder mangelhafte Studienbedingungen? Die Hochschule: Journal für Wissenschaft und Bildung, 20(1), 110-126.

Blüthmann, I. (2012). Individuelle und studienbezogene Einflussfaktoren auf die Zufriedenheit von Bachelorstudierenden. Zeitschrift für Erziehungswissenschaft, 15(2), 273 - 303. https://doi. org/10.1007/s11618-012-0270-3

Carstensen, B. \& Klusmann, U. (2021). Assertiveness and adaptation: Prospective teachers' social competence development and its significance for occupational well-being. The British Journal of Educational Psychology, 91(1), 500 - 526. https://doi. org/10.1111/bjep.12377

Carstensen, B., Lindner, C., \& Klusmann, U. (2021). Satisfaction, enthusiasm, and well-being of student teachers: A question of appreciation? PsychArchives. Verfügbar unter https://doi.org/ 10.23668/PSYCHARCHIVES.4564

Deuer, E. (2017). Gratifikationskrisen im Kontext des dualen Studiums: Ursachen, Ausmaß und mögliche Konsequenzen. Stuttgart: Arbeitspapiere zur Hochschulforschung, 5 / 2017.

Diener, E., Suh, E. M., Lucas, R. E., \& Smith, H. L. (1999). Subjective well-being: Three decades of progress. Psychological Bulletin, 125(2), $276-302$.

Enders, C. K. (2010). Applied missing data analysis. Methodology in the social sciences. New York: Guilford Press.

Enzmann, D. \& Kleiber, D. (1989). Helfer-Leiden: Stress und Burnout in psychosozialen Berufen. Heidelberg: Asanger.
Erdmann, M. \& Ratzlaff, O. (2017). Wahrnehmung und Heterogenität von Fach- und Lehramtsstudierenden im Kontext von Lehrveranstaltungen. Potsdamer Beiträge zur Hochschulforschung, 3, $181-196$.

Ertl, B., Luttenberger, S. \& Paechter, M. (2014). Stereotype als Einflussfaktoren auf die Motivation und die Einschätzung der eigenen Fähigkeiten bei Studentinnen in MINT-Fächern. Gruppendynamik und Organisationsberatung, 45(4), 419-440. https://doi.org/10.1007/s11612-014-0261-3

Eurydice (2004). Der Lehrerberuf in Europa: Profil, Tendenzen und Anliegen. Bericht IV: Die Attraktivität des Lehrerberufs im 21. Jahrhundert. Allgemein bildender Sekundarbereich I. Brüssel: Eurydice.

Fellenberg, F. \& Hannover, B. (2006). Kaum begonnen, schon zerronnen? Psychologische Ursachenfaktoren für die Neigung von Studienanfängern, das Studium abzubrechen oder das Fach zu wechseln. Empirische Pädagogik, 20(4), 381 - 399.

Fiske, S. T., Cuddy, A. J., Glick, P., \& Xu, J. (2002). A model of (often mixed) stereotype content: Competence and warmth respectively follow from perceived status and competition. Journal of Personality and Social Psychology, 82(6), 878-902.

Frenzel, A. C., Goetz, T., Lüdtke, O., Pekrun, R. \& Sutton, R. E. (2009). Emotional transmission in the classroom: Exploring the relationship between teacher and student enjoyment. Journal of Educational Psychology, 101(3), 705-716. https://doi.org/10. 1037/a0014695

Gavish, B. \& Friedman, I. A. (2010). Novice teachers' experience of teaching: A dynamic aspect of burnout. Social Psychology of Education, 13(2), 141-167. https://doi.org/10.1007/s11218009-9108-0

Gómez Tutor, C., Hobelsberger, C. \& Menzer, C. (2011). Zwischen Serviceanspruch und Eigenverantwortung - ein Balanceakt in Zeiten Bolognas. Zeitschrift für Hochschulentwicklung, 6(2), $238-249$.

Günther, E. A. \& Koeszegi, S. T. (2015). „Das ist aber nicht der akademische Gedanke" - Ansprüche an Lehrende und von Lehrenden einer Technischen Universität. In K. Rheinländer (Hrsg.), Ungleichheitssensible Hochschullehre: Positionen, Voraussetzungen, Perspektiven (S.141-163). Wiesbaden: Springer VS.

Halbesleben, J. R. B. (2006). Sources of social support and burnout: A meta-analytic test of the conservation of resources model. The Journal of Applied Psychology, 91(5), 1134-1145. https:// doi.org/10.1037/0021-9010.91.5.1134

Ihme, T. A. \& Möller, J. (2015). „He who can, does; he who cannot, teaches?" Stereotype threat and preservice teachers. Journal of Educational Psychology, 107(1), 300 -308. https://doi.org/10. 1037/a0037373

Isleib, S., Woisch, A. \& Heublein, U. (2019). Ursachen des Studienabbruchs: Theoretische Basis und empirische Faktoren. Zeitschrift für Erziehungswissenschaft, 22(5), 1047 - 1076. https:// doi.org/10.1007/s11618-019-00908-x

Kinman, G., Wray, S. \& Strange, C. (2011). Emotional labour, burnout and job satisfaction in UK teachers: The role of workplace social support. Educational Psychology, 31(7), 843 - 856. https:// doi.org/10.1080/01443410.2011.608650

Klemm, K. (2015). Lehrerinnen und Lehrer der MINT-Fächer: Zur Bedarfs- und Angebotsentwicklung in den allgemein bildenden Schulen der Sekundarstufen I und II am Beispiel NordrheinWestfalens. Gutachten im Auftrag der Deutsche Telekom Stiftung.

Kultusministerkonferenz [KMK] (2020). Lehrereinstellungsbedarf und-angebot in der Bundesrepublik Deutschland 2020-2030 Zusammengefasste Modellrechnungen der Länder.

Kunter, M., Frenzel, A., Nagy, G., Baumert, J., \& Pekrun, R. (2011). Teacher enthusiasm: Dimensionality and context specificity. Contemporary Educational Psychology, 36(4), 289 - 301. 
Kunter, M., Tsai, Y.-M., Klusmann, U., Brunner, M., Krauss, S. \& Baumert, J. (2008). Students' and mathematics teachers' perceptions of teacher enthusiasm and instruction. Learning and Instruction, 18(5), 468-482. https://doi.org/10.1016/j.learnin struc.2008.06.008

Lindner, C. \& Klusmann, U. (2018). Fachwissenschaftliche und fachdidaktische Inhalte der Lehramtsausbildung. Empirische Evidenz für die Notwendigkeit einer integrativen Vernetzung. In Brouer, B., Burda-Zoyke, A., Kilian, J. \& Petersen, I. (Hrsg.), Vernetzung in der Lehrerinnen- und Lehrerbildung. Ansätze, Methoden und erste Befunde aus dem LeaP-Projekt an der ChristianAlbrechts-Universität zu Kiel (S.287 - 298). Münster: Waxmann.

Lindner, C., Klusmann, U., Baum, M., Brouër, B., Burda-Zoyke, A., Heinz, T. et al. (2018). STePS 2018: Skalenhandbuch zur Dokumentation der Evaluationsinstrumente im Projekt „Lehramt mit Perspektive an der CAU Kiel“" - 2. Messzeitpunkt. Kiel: IPN.

Lindner, C., Klusmann, U., Baum, M., Brouër, B., Heinz, T., Kilian, J. et al. (2018). STePS 2017: Skalenhandbuch zur Dokumentation der Evaluationsinstrumente im Projekt „Lehramt mit Perspektive an der CAU Kiel“ - 1. Messzeitpunkt. Kiel: IPN.

Mäkinen, J., Olkinuora, E. \& Lonka, K. (2004). Students at risk: Students' general study orientations and abandoning/prolonging the course of studies. Higher Education, 48, 173-188.

Maslach, C., Jackson, S. E. \& Leiter, M. P. (1996). Maslach Burnout Inventory Manual. Palo Alto, California.

Münst, S. A. (2008). Hierarchie, Fachkompetenz und Geschlecht in Lehrveranstaltungen: Ergebnisse einer ethnographischen Teilnehmenden Beobachtung. In K. Zimmermann, M. Kamphans \& S. Metz-Göckel (Hrsg.), Perspektiven der Hochschulforschung (S.179 - 196). Wiesbaden: VS Verlag für Sozialwissenschaften.

Muthén, L. K., Muthén, B. O. (1998-2015). Mplus user's guide (version 7.4). Los Angeles, CA: Muthén \& Muthén.

Neugebauer, M., Heublein, U. \& Daniel, A. (2019). Studienabbruch in Deutschland: Ausmaß, Ursachen, Folgen, Präventionsmöglichkeiten. Zeitschrift für Erziehungswissenschaft, 22(5), 1025 - 1046. https://doi.org/10.1007/s11618-019-00904-1

Nezlek, J. B., Schröder-Abé, M. \& Schütz, A. (2006). Mehrebenenanalysen in der psychologischen Forschung. Psychologische Rundschau, 57(4), 213-223. https://doi.org/10.1026/00333042.57.4.213

Pfister, I. B., Jacobshagen, N., Kälin, W., Stocker, D., Meier, L. L. \& Semmer, N. K. (2020). Appreciation and illegitimate tasks as predictors of affective well-being: Disentangling within- and between-person effects. Journal of Work and Organizational Psychology, 36(1), 63 - 75. https://doi.org/10.5093/jwop2020a6

Pinn, S. \& Rothland, M. (2011). Die „Anti-Elite“ - dumm, neurotisch und falsch motiviert? Das Bild angehender Lehrkräfte in den Printmedien. Seminar, 17(4), $136-148$.

Renger, D., Renger, S., Miché, M. \& Simon, B. (2017). A social recognition approach to autonomy: The role of equality-based respect. Personality and Social Psychology Bulletin, 43(4), 479 - 492. https://doi.org/10.1177/0146167216688212

Roloff Henoch, J., Klusmann, U., Lüdtke, O. \& Trautwein, U. (2015). Who becomes a teacher? Challenging the "negative selection" hypothesis. Learning and Instruction, 36, 46-56. https://doi. org/10.1016/j.learninstruc.2014.11.005

Rothland, M. (2016). Der Lehrerberuf in der Öffentlichkeit. In M. Rothland (Hrsg.), Beruf Lehrer/Lehrerin. Ein Studienbuch (S.67-86). Münster: Waxmann.
Schneider, M. \& Preckel, F. (2017). Variables associated with achievement in higher education: A systematic review of metaanalyses. Psychological Bulletin, 143(6), 565-600. https://doi. org/10.1037/bul0000098

Siegrist, J. (1996). Adverse health effects of high-effort/low-reward conditions. Journal of Occupational Health Psychology, 1(1), $27-41$.

Stocker, D., Jacobshagen, N., Krings, R., Pfister, I. B. \& Semmer, N. K. (2014). Appreciative leadership and employee well-being in everyday working life. Zeitschrift für Personalforschung, 28(1-2), $73-95$.

Stocker, D., Jacobshagen, N., Semmer, N. K. \& Annen, H. (2010). Appreciation at work in the Swiss armed forces. Swiss Journal of Psychology, 69(2), 117-124.

Stocker, D., Keller, A. C., Meier, L. L., Elfering, A., Pfister, I. B., Jacobshagen, N. \& Semmer, N. K. (2019). Appreciation by supervisors buffers the impact of work interruptions on well-being longitudinally. International Journal of Stress Management, 26(4), 331 - 343. https://doi.org/10.1037/str0000111

Trapmann, S., Hell, B., Weigand, S. \& Schuler, H. (2007). Die Validität von Schulnoten zur Vorhersage des Studienerfolgs - eine Metaanalyse. Zeitschrift für Pädagogische Psychologie, 21,11-27.

Van Quaquebeke, N. \& Felps, W. (2016). Respectful inquiry: A motivational account of leading through asking open questions and listening. Academy of Management Review, 43, 1- 23.

Viswesvaran, C., Sanchez, J. I. \& Fisher, J. (1999). The role of social support in the process of work stress: A meta-analysis. Journal of Vocational Behavior, 54(2), 314-334. https://doi.org/10.1006/ jvbe.1998.1661

Westermann, R., Elke, H., Spies, K. \& Trautwein, U. (1996). Identifikation und Erfassung von Komponenten der Studienzufriedenheit. Psychologie in Erziehung und Unterricht, 43(1), 1 - 22.

Yukl, G., Gordon, A. \& Taber, T. (2002). A hierarchical taxonomy of leadership behavior: Integrating a half century of behavior research. Journal of Leadership and Organizational Studies, 9(1), $15-32$.

\section{Historie}

Manuskript eingereicht: 14.04.2021

Manuskript nach Revision angenommen: 19.11.2021

Onlineveröffentlichung: 03.12.2021

\section{Förderung}

IPN - Leibniz-Institut für die Pädagogik der Naturwissenschaften und Mathematik, Kiel.

\section{ORCID}

Bastian Carstensen

(iD) https://orcid.org/0000-0001-5259-9578

\section{Dr. Bastian Carstensen}

IPN - Leibniz-Institut für die Pädagogik der Naturwissenschaften und Mathematik

Erziehungswissenschaft und Pädagogische Psychologie

Olshausenstraße 62

24118 Kiel

Deutschland

bcarstensen@leibniz-ipn.de 